\title{
NEGATIVE POWER NONLINEAR INTEGRAL EQUATIONS ON BOUNDED DOMAINS
}

\author{
DEDICATE TO HAÏM BREZIS ON OCCASION OF HIS 75TH BIRTHDAY
}

JINGBO DOU, QIANQIAO GUO AND MEIJUN ZHU

\begin{abstract}
This is the continuation of our previous work [5], where we introduced and studied some nonlinear integral equations on bounded domains that are related to the sharp Hardy-Littlewood-Sobolev inequality. In this paper, we introduce some nonlinear integral equations on bounded domains that are related to the sharp reversed Hardy-Littlewood-Sobolev inequality. These are integral equations with nonlinear term involving negative exponents. Existence results as well as nonexistence results are obtained.
\end{abstract}

Keywords Reversed sharp Hardy-Littlewood-Sobolev inequality, Integral equations, Existence, nonexistence

Mathematics Subject Classification(2000). 45G10, 35J60

\section{Introduction}

In [5, motivated by the study of certain semi-linear equations and the sharp Sobolev inequality, we introduced and studied the integral equations (with positive power) related to the sharp Hardy-Littlewood-Sobolev (HLS for short) inequality. Let us briefly recall these as the follows.

For $0<\alpha<n$, on any bounded domain $\Omega \subset \mathbb{R}^{n}$ with smooth boundary, we considered

$$
\hat{\xi}_{\alpha}(\Omega)=\sup _{f \in L^{\frac{2 n}{n+\alpha}}(\Omega), f \neq 0} \frac{\int_{\Omega} \int_{\Omega} f(x)|x-y|^{-(n-\alpha)} f(y) d x d y}{\|f\|_{L^{\frac{2 n}{n+\alpha}}(\Omega)}^{2}} .
$$

It was showed in [5] that $\hat{\xi}_{\alpha}(\Omega)=N_{\alpha}$, where $N_{\alpha}$ is the best constant of the classical sharp HLS inequality (due to Lieb [7]); And $\hat{\xi}_{\alpha}(\Omega)$ is not attained by any functions if $\Omega \neq \mathbb{R}^{n}$. This indicates that there is not any energy maximizing solution to

$$
f^{\frac{n-\alpha}{n+\alpha}}(x)=\int_{\Omega} \frac{f(y)}{|x-y|^{n-\alpha}} d y, \quad f \geq 0, \quad x \in \bar{\Omega} .
$$

We then considered a general integral equation

$$
f^{q-1}(x)=\int_{\Omega} \frac{f(y)}{|x-y|^{n-\alpha}} d y+\lambda \int_{\Omega} \frac{f(y)}{|x-y|^{n-\alpha-1}} d y, \quad f \geq 0, \quad x \in \bar{\Omega},
$$

for $\alpha<n$, and studied the existence and nonexistence of positive solutions for different power $q$ and parameter $\lambda$.

In this paper we consider integral equation (1.1) for $\alpha>n$. This case is related to so called sharp reversed HLS inequality, which was discovered by Dou and Zhu [4].

Recall the sharp reversed HLS inequality from [4] (see also related work by Ngô, Nguyen [8] and by Beckner [1]): 
Theorem A. For $\alpha>n$,

$$
\left|\int_{\mathbb{R}^{n}} \int_{\mathbb{R}^{n}} f(x)\right| x-\left.y\right|^{-(n-\alpha)} g(y) d x d y \mid \geq N_{\alpha}\|f\|_{L^{\frac{2 n}{n+\alpha}\left(\mathbb{R}^{n}\right)}}\|g\|_{L^{\frac{2 n}{n+\alpha}\left(\mathbb{R}^{n}\right)}}
$$

holds for all non-negative functions $f, g \in L^{\frac{2 n}{n+\alpha}}\left(\mathbb{R}^{n}\right)$, where

$$
N_{\alpha}=N\left(\frac{2 n}{n+\alpha}, \alpha, n\right)=\pi^{(n-\alpha) / 2} \frac{\Gamma(\alpha / 2)}{\Gamma(n / 2+\alpha / 2)}\left\{\frac{\Gamma(n / 2)}{\Gamma(n)}\right\}^{-\alpha / n}
$$

And the equality holds if and only if

$$
f=c_{1} g=c_{2}\left(\frac{1}{c_{3}+\left|x-x_{0}\right|^{2}}\right)^{\frac{n+\alpha}{2}},
$$

where $c_{1}, c_{2}, c_{3}$ are any positive constants, and $x_{0} \in \mathbb{R}^{n}$.

Similar to what is done in [5]: for any smooth domain $\Omega \subset \mathbb{R}^{n}$, we consider

$$
\xi_{\alpha}(\Omega)=\inf _{f \in L^{\frac{2 n}{n+\alpha}}(\Omega), f \geq 0, f \neq 0} \frac{\int_{\Omega} \int_{\Omega} f(x)|x-y|^{-(n-\alpha)} f(y) d x d y}{\|f\|_{L^{\frac{2 n}{n+\alpha}}(\Omega)}^{2}} .
$$

We will show that

$$
\xi_{\alpha}(\Omega)=N_{\alpha},
$$

and $\xi_{\alpha}(\Omega)$ is not attained by any functions if $\Omega \neq \mathbb{R}^{n}$ (see Proposition 2.1 below). Again, we notice that the corresponding Euler-Lagrange equation for the minimizer (if the minimum is attained) is integral equation (1.1) with $\lambda=0$ and a negative power $\left(\frac{n-\alpha}{n+\alpha}<0\right)$. We thus know that if $\alpha>n$ there is no energy minimizing solution to integral equation (1.1) for $q=2 n /(n+\alpha)$ and $\lambda=0$.

Let $p_{\alpha}=2 n /(n-\alpha), q_{\alpha}=2 n /(n+\alpha)$ and $d(\Omega)=\sup _{x, y \in \Omega}|x-y|$ be the diameter of the bounded domain $\Omega$. In this paper, we consider integral equation (1.1) for $\alpha>n$. We shall prove

Theorem 1.1. Assume $\alpha>n$ and $\Omega$ is a bounded domain with smooth boundary. (1) For $0<q<q_{\alpha}$ (subcritical case), and $-\frac{1}{d(\Omega)}<\lambda$, there is a positive solution $f \in C^{1}(\bar{\Omega})$ to equation (1.1).

(2) For $q=q_{\alpha}$ (critical case), and $-\frac{1}{d(\Omega)}<\lambda<0$, there is a positive solution $f \in C^{1}(\bar{\Omega})$ to equation (1.1).

(3) For $q_{\alpha} \leq q<1$ (critical and supercritical cases), and $\lambda \geq 0$, if $\Omega$ is a star-shaped domain, then there is not any positive $C^{1}(\bar{\Omega})$ solution to (1.1).

We emphasis here that $q_{\alpha}<1$ (since $\alpha>n$ ). Thus equation (1.1) has a nonlinear term with a negative power. Our results indicate that even though the integral equation, which is related to the reversed HLS inequality, is of negative nonlinearity, similar phenomena to the integral equation with positive power can be seen. Contrary to integral equations with positive nonlinearity, it seems that no compact embedding can be used directly for the existence result to the integral equation with subcritical negative power. Different techniques are needed for deriving the existence as well as the nonexistence results. See more details in Section 3 below.

We organize the paper as follows: In Section 2, we focus on the nonexistence result (part (3) of Theorem 1.1). In Section 3, we first obtain the existence result (part (1) of Theorem 1.1), and then we show the symmetric and monotonically increasing properties of solutions to the integral equation on a ball, even no pointwise 
boundary condition is given (Theorem 3.3 below). In Section 4, we come back to the integral equations with critical exponent and a lower order term and prove the existence result (part (2) of Theorem 1.1).

Notation: for any function $f(x)$ defined on $\Omega$, we always use $\tilde{f}(x)$ to represent its trivial extension in $\mathbb{R}^{n}$, namely,

$$
\tilde{f}(x):= \begin{cases}f(x) & x \in \Omega \\ 0 & x \in \mathbb{R}^{n} \backslash \Omega .\end{cases}
$$

We also denote

$$
I_{\alpha} f(x):=\int_{\mathbb{R}^{n}} \frac{f(y)}{|x-y|^{n-\alpha}} d y, \quad I_{\alpha, \Omega} f(x):=\int_{\Omega} \frac{f(y)}{|x-y|^{n-\alpha}} d y
$$

and

$$
L_{+}^{q}(\Omega):=\left\{f \in L^{q}(\Omega) \backslash\{0\}: f \geq 0\right\} .
$$

\section{NONEXISTENCE FOR CRITICAL AND SUPERCRITICAL CASES}

In this section, we first derive energy estimate (1.5) for any domain $\Omega \subset \mathbb{R}^{n}$, and then show that the infimum $\xi_{\alpha}(\Omega)$ is not achieved by any function once $\Omega \neq \mathbb{R}^{n}$.

Proposition 2.1. For any domain $\Omega \subset \mathbb{R}^{n}, \xi_{\alpha}(\Omega)=N_{\alpha}$. Further, if $\Omega \neq \mathbb{R}^{n}$, then the infimum $\xi_{\alpha}(\Omega)$ is not achieved by any function in $L^{q_{\alpha}}(\Omega)$.

Proof. If $f \in L_{+}^{q_{\alpha}}(\Omega)$, then $\tilde{f} \in L_{+}^{q_{\alpha}}\left(\mathbb{R}^{n}\right)$. It follows that

$$
\begin{aligned}
\xi_{\alpha}(\Omega) & =\inf _{f \in L_{+}^{q_{\alpha}}(\Omega) \backslash\{0\}} \frac{\int_{\mathbb{R}^{n}} \int_{\mathbb{R}^{n}} \tilde{f}(x)|x-y|^{-(n-\alpha)} \tilde{f}(y) d x d y}{\|\tilde{f}\|_{L^{q_{\alpha}}\left(\mathbb{R}^{n}\right)}^{2}} \\
& \geq \inf _{g \in L_{+}^{q_{\alpha}}\left(\mathbb{R}^{n}\right) \backslash\{0\}} \frac{\int_{\mathbb{R}^{n} \int_{\mathbb{R}^{n}} g(x)|x-y|^{-(n-\alpha)} g(y) d x d y}}{\|g\|_{L^{q_{\alpha}\left(\mathbb{R}^{n}\right)}}^{2}}=N_{\alpha} .
\end{aligned}
$$

On the other hand, recall that $f(x)=\left(\frac{1}{1+|x|^{2}}\right)^{\frac{n+\alpha}{2}}$ is an extremal function to the sharp reversed HLS inequality in Theorem A, as well as its conformal equivalent class:

$$
f_{\epsilon}(x)=\epsilon^{-\frac{n+\alpha}{2}} f\left(\frac{\left|x-x_{*}\right|}{\epsilon}\right)=\left(\frac{\epsilon}{\epsilon^{2}+\left|x-x_{*}\right|^{2}}\right)^{\frac{n+\alpha}{2}},
$$

where $x_{*} \in \mathbb{R}^{n}, \epsilon>0$. Thus

$$
\left\|I_{\alpha} f\right\|_{L^{p_{\alpha}\left(\mathbb{R}^{n}\right)}}=\left\|I_{\alpha} f_{\epsilon}\right\|_{L^{p_{\alpha}\left(\mathbb{R}^{n}\right)}}, \quad\|f\|_{L^{q_{\alpha}\left(\mathbb{R}^{n}\right)}}=\left\|f_{\epsilon}\right\|_{L^{q_{\alpha}\left(\mathbb{R}^{n}\right)}} .
$$

Choose $x_{0}=x_{*}$ for some point $x_{0} \in \Omega$ and $R$ small enough so that $B_{R}\left(x_{0}\right) \subset \Omega$. Then we define test function $g(x)$ as

$$
g(x)= \begin{cases}f_{\epsilon}(x) & x \in B_{R}\left(x_{0}\right) \subset \Omega, \\ 0 & x \in \mathbb{R}^{n} \backslash B_{R}\left(x_{0}\right) .\end{cases}
$$


Obviously, $g \in L_{+}^{q_{\alpha}}\left(\mathbb{R}^{n}\right)$. Thus,

$$
\begin{aligned}
& \int_{\Omega} \int_{\Omega} \frac{g(x) g(y)}{|x-y|^{n-\alpha}} d x d y \\
= & \int_{\mathbb{R}^{n}} \int_{\mathbb{R}^{n}} \frac{f_{\epsilon}(x) f_{\epsilon}(y)}{|x-y|^{n-\alpha}} d x d y-2 \int_{\mathbb{R}^{n}} \int_{\mathbb{R}^{n} \backslash B_{R}\left(x_{0}\right)} \frac{f_{\epsilon}(x) f_{\epsilon}(y)}{|x-y|^{n-\alpha}} d x d y \\
& +\int_{\mathbb{R}^{n} \backslash B_{R}\left(x_{0}\right)} \int_{\mathbb{R}^{n} \backslash B_{R}\left(x_{0}\right)} \frac{f_{\epsilon}(x) f_{\epsilon}(y)}{|x-y|^{n-\alpha}} d x d y \\
\leq & N_{\alpha}\left\|f_{\epsilon}\right\|_{L^{q_{\alpha}\left(\mathbb{R}^{n}\right)}-2 I_{1},}^{2}
\end{aligned}
$$

where

$$
I_{1}:=\int_{\mathbb{R}^{n}} \int_{\mathbb{R}^{n} \backslash B_{R}\left(x_{0}\right)} \frac{f_{\epsilon}(x) f_{\epsilon}(y)}{|x-y|^{n-\alpha}} d x d y .
$$

Notice that $f_{\epsilon}(x)$ is an extremal function for the sharp reversed HLS inequality. Thus it satisfies integral equation:

$$
f^{\frac{n-\alpha}{n+\alpha}}(x)=B \int_{\mathbb{R}^{n}} \frac{f(y)}{|x-y|^{n-\alpha}} d y,
$$

where $B$ is a suitable positive constant. We thus can estimate $I_{1}$ in the following:

$$
I_{1}=C \int_{\mathbb{R}^{n} \backslash B_{R}\left(x_{0}\right)} f_{\epsilon}^{\frac{2 n}{n+\alpha}}(x) d x=O\left(\frac{R}{\epsilon}\right)^{-n}, \quad \text { as } \quad \epsilon \rightarrow 0 .
$$

And we also have

$$
\begin{aligned}
\int_{B_{R}\left(x_{0}\right)} f_{\epsilon}^{\frac{2 n}{n+\alpha}}(x) d x & =\int_{\mathbb{R}^{n}} f_{\epsilon}^{\frac{2 n}{n+\alpha}}(x) d x-\int_{\mathbb{R}^{n} \backslash B_{R}\left(x_{0}\right)} f_{\epsilon}^{\frac{2 n}{n+\alpha}}(x) d x \\
& =\int_{\mathbb{R}^{n}} f_{\epsilon}^{\frac{2 n}{n+\alpha}}(x) d x-O\left(\frac{R}{\epsilon}\right)^{-n}, \quad \text { as } \quad \epsilon \rightarrow 0 .
\end{aligned}
$$

Hence, for small enough $\epsilon>0$, we have

$$
\begin{aligned}
\xi_{\alpha}(\Omega) & \leq \frac{\int_{\Omega} \int_{\Omega} \frac{g(x) g(y)}{|x-y|^{n-\alpha}} d x d y}{\|g\|_{L^{q_{\alpha}(\Omega)}}^{2}} \\
& \leq \frac{N_{\alpha}\left\|f_{\epsilon}\right\|_{L^{q_{\alpha}\left(\mathbb{R}^{n}\right)}}^{2}-I_{1}}{\left\|f_{\epsilon}\right\|_{L^{q_{\alpha}\left(B_{R}\left(x_{0}\right)\right)}}^{2}} \\
& =\frac{N_{\alpha}\left\|f_{\epsilon}\right\|_{L^{q_{\alpha}\left(\mathbb{R}^{n}\right)}}^{2}-O\left(\frac{R}{\epsilon}\right)^{-n}}{\left\|f_{\epsilon}\right\|_{L^{q_{\alpha}\left(\mathbb{R}^{n}\right)}}^{2}-O\left(\frac{R}{\epsilon}\right)^{-n}},
\end{aligned}
$$

which yields $\xi_{\alpha}(\Omega) \leq N_{\alpha}$ as $\epsilon \rightarrow 0$.

Finally, we show that $\xi_{\alpha}(\Omega)$ is not achieved if $\Omega \neq \mathbb{R}^{n}$. In fact, if $\xi_{\alpha}(\Omega)$ were attained by some function $u \in L_{+}^{q_{\alpha}}(\Omega)$, then $\tilde{u} \in L_{+}^{q_{\alpha}}\left(\mathbb{R}^{n}\right)$ would be an extremal function to the sharp reversed HLS inequality on $\mathbb{R}^{n}$, which is impossible due to Theorem A.

Proposition 2.1 indicates that for $\alpha>n$ there is not any minimizing energy solution to (1.1) for $q=2 n /(n+\alpha)$ and $\lambda=0$. In fact, we will show that there is not any positive $C^{1}$ solution to (1.1) for $\alpha>n, q=2 n /(n+\alpha)$ and $\lambda=0$ on any star-shaped domain. 
Proof of part (3) in Theorem 1.1 (nonexistence part). Without loss of generality, here we assume that the origin is in $\Omega$ and the domain is star-shaped with respect to the origin.

Recall the following Pohozaev identity from [5].

Lemma 2.2. Assume that the origin is in $\Omega$ and the domain is star-shaped with respect to the origin. If $u \in C^{1}(\bar{\Omega})$ is a non-negative solution to

$$
u(x)=\int_{\Omega} \frac{u^{p-1}(y)}{|x-y|^{n-\alpha}} d y+\lambda \int_{\Omega} \frac{u^{p-1}(y)}{|x-y|^{n-\alpha-1}} d y, \quad x \in \bar{\Omega},
$$

where $p \neq 0, \lambda \in \mathbb{R}$, then

$\left(\frac{n}{p}+\frac{\alpha-n}{2}\right) \int_{\Omega} u^{p}(x) d x=-\frac{\lambda}{2} \int_{\Omega} \int_{\Omega} \frac{u^{p-1}(x) u^{p-1}(y)}{|x-y|^{n-\alpha}} d y d x+\frac{1}{p} \int_{\partial \Omega}(x \cdot \nu) u^{p}(x) d \sigma,(2$

where $\nu$ is the outward unit normal vector to $\partial \Omega$.

Applying Lemma 2.2 to (1.1) for $\lambda \geq 0$ and $u(x)=f^{q-1}(x)$. Thus $p=\frac{q}{q-1}=q^{\prime}$. Noticing that $1>q \geq q_{\alpha}$ is equivalent to $p \leq p_{\alpha}<0$, we have

$$
-\frac{\lambda}{2} \int_{\Omega} \int_{\Omega} \frac{u^{p-1}(x) u^{p-1}(y)}{|x-y|^{n-\alpha-1}} d y d x+\frac{1}{p} \int_{\partial \Omega}(x \cdot \nu) u^{p-1}(x) d \sigma \geq 0 .
$$

Since $\Omega$ is star-shaped domain about the origin, we have $x \cdot \nu>0$ on $\partial \Omega$. If $\lambda>0$, then $u(x) \equiv \infty$ on $\bar{\Omega}$. If $\lambda=0$, then $u \equiv \infty$ on $\partial \Omega$. Therefore we obtain a contradiction to that $f(x)$ is a positive $C^{1}(\bar{\Omega})$ solution.

Remark 2.3. Condition $q<1$ is needed in our proof. If $q>1$, one may call it a supercritical exponent (since it is bigger than $q_{\alpha}$ ). However, in this case $p=$ $q /(q-1)>0$ is also bigger than negative $p_{\alpha}$. Our nonexistence result may not be true any more in this case.

Note that the unit ball is conformally equivalent to the upper half space. We have

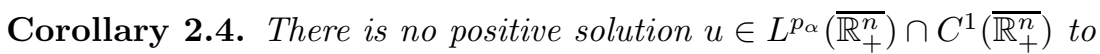

$$
u(x)=\int_{\mathbb{R}_{+}^{n}} \frac{u^{p_{\alpha}-1}(y)}{|x-y|^{n-\alpha}} d y, \quad x \in \overline{\mathbb{R}_{+}^{n}} .
$$

\section{EXISTENCE RESUlt FOR SUbCRITICAL CASE}

For subcritical exponents we have the following inequality:

Lemma 3.1. Let $q \in\left(0, q_{\alpha}\right)$. There exists a positive constant $C(n, q, \alpha, \Omega)>0$ such that

$$
\int_{\Omega} \int_{\Omega} f(x)|x-y|^{-(n-\alpha)} f(y) d x d y \geq C(n, q, \alpha, \Omega)\|f\|_{L^{q}(\Omega)}^{2}
$$

holds for any non-negative function $f \in L^{q}(\Omega)$.

Proof. For $f \in L^{q}(\Omega)$, by using the reversed HLS inequality (1.2) we have

$$
\begin{aligned}
\left\langle I_{\alpha, \Omega} f, f\right\rangle & =\int_{\mathbb{R}^{n}} \int_{\mathbb{R}^{n}} \tilde{f}(x)|x-y|^{-(n-\alpha)} \tilde{f}(y) d x d y \\
& \geq N_{\alpha}\|\tilde{f}\|_{L^{q_{\alpha}\left(\mathbb{R}^{n}\right)}}^{2}=N_{\alpha}\|\tilde{f}\|_{L^{q_{\alpha}(\Omega)}}^{2} \\
& \geq C(n, q, \alpha, \Omega)\|f\|_{L^{q}(\Omega)}^{2} .
\end{aligned}
$$


We would like to point out that one can also prove the above lemma directly via a Young type inequality as that in Dou, Guo and Zhu [6].

Based on the above lemma, we can obtain the existence result for subcritical exponent (part (1) of Theorem 1.1). Notice that it is different from the case $0<$ $\alpha<n$ (Lemma 3.2 in [5]) since no compact embedding can be used directly here. We follow a similar approach used in Dou, Guo and Zhu [6].

Lemma 3.2. For $0<q<q_{\alpha}, \lambda>-\frac{1}{d(\Omega)}$, infimum

$\xi_{\alpha, q}(\Omega):=\inf _{f \in L_{+}^{q}(\Omega)} \frac{\int_{\Omega} \int_{\Omega}\left(f(x)|x-y|^{-(n-\alpha)} f(y)+\lambda f(x)|x-y|^{-(n-\alpha-1)} f(y)\right) d y d x}{\|f\|_{L^{q}(\Omega)}^{2}}>0$,

and it is attained by some nonnegative function in $L_{+}^{q}(\Omega)$.

Proof. Notice: for $x, y \in \Omega$ and $0>\lambda>-1 / d(\Omega), 1+\lambda|x-y| \geq 1+\lambda d(\Omega)>0$. Thus we know $\xi_{\alpha, q}(\Omega)>0$ by Lemma 3.1 .

Choose a minimizing nonnegative sequence $\left\{f_{j}\right\}_{j=1}^{\infty}$ in $L^{q}(\Omega)$. Assume without loss of generality that $f_{j} \in L^{q_{\alpha}}(\Omega)$ (see, for example, Proposition 2.5 in [6]). Then we can normalize it such that $\left\|f_{j}\right\|_{L^{q_{\alpha}(\Omega)}}=1$. It follows that there exists a subsequence such that

$$
f_{j}^{q} \rightarrow f_{*}^{q} \quad \text { weakly in } \quad L^{\frac{q_{\alpha}}{q}}(\Omega), \text { as } j \rightarrow \infty .
$$

Then

$$
\int_{\Omega} f_{j}^{q} \rightarrow \int_{\Omega} f_{*}^{q}, \text { as } j \rightarrow \infty
$$

Claim: $\left\|f_{j}\right\|_{L^{1}(\Omega)} \leq C$.

We relegate the proof of this claim to the end.

Once the claim is proved, we have $\int_{\Omega} f_{*}^{q}>C>0$ via an interpolation inequality and $f_{j}^{q} \rightarrow f_{*}^{q} \quad$ weakly in $\quad L^{\frac{1}{q}}(\Omega)$. Then for any fixed $x \in \bar{\Omega}, f_{*}^{1-q}(y)|x-y|^{\alpha-n}(1+$ $\lambda|x-y|) \in L^{\frac{1}{1-q}}(\Omega)$, thus, as $j \rightarrow \infty$,

$$
\int_{\Omega} f_{j}^{q}(y) f_{*}^{1-q}(y)|x-y|^{\alpha-n}(1+\lambda|x-y|) d y \rightarrow \int_{\Omega} f_{*}(y)|x-y|^{\alpha-n}(1+\lambda|x-y|) d y .
$$

Further, we show that the above convergence is actually uniformly convergent for all $x \in \bar{\Omega}$.

By Hölder's inequality we have

$$
\begin{aligned}
& \int_{\Omega} f_{j}^{q}(y) f_{*}^{1-q}(y)|x-y|^{\alpha-n}(1+\lambda|x-y|) d y \\
\leq & \left(\int_{\Omega} f_{j}^{q \cdot \frac{1}{q}}(y) d y\right)^{q}\left(\int_{\Omega}\left(f_{*}^{1-q}(y)|x-y|^{\alpha-n}(1+\lambda|x-y|)\right)^{\frac{1}{1-q}} d y\right)^{1-q} \leq C,
\end{aligned}
$$

that is, $\int_{\Omega} f_{j}^{q}(y) f_{*}^{1-q}(y)|x-y|^{\alpha-n}(1+\lambda|x-y|) d y$ is uniformly bounded for $x \in \bar{\Omega}$. Notice that for any $x_{1}, x_{2}, y \in \bar{\Omega}$,

$$
|| x_{1}-\left.y\right|^{\alpha-n}-\left|x_{2}-y\right|^{\alpha-n} \mid \leq\left\{\begin{array}{cl}
C\left|x_{1}-x_{2}\right|^{\alpha-n}, & \text { if } 0<\alpha-n \leq 1 \\
C\left|x_{1}-x_{2}\right|, & \text { if } \alpha-n>1 \\
6 &
\end{array}\right.
$$


Then for any $x_{1}, x_{2} \in \bar{\Omega}$,

$$
\begin{aligned}
& \left|\int_{\Omega} f_{j}^{q}(y) f_{*}^{1-q}(y)\right| x_{1}-\left.y\right|^{\alpha-n}\left(1+\lambda\left|x_{1}-y\right|\right) d y \\
& -\int_{\Omega} f_{j}^{q}(y) f_{*}^{1-q}(y)\left|x_{2}-y\right|^{\alpha-n}\left(1+\lambda\left|x_{2}-y\right|\right) d y \mid \\
\leq & \int_{\Omega} f_{j}^{q}(y) f_{*}^{1-q}(y)|| x_{1}-\left.y\right|^{\alpha-n}\left(1+\lambda\left|x_{1}-y\right|\right)-\left|x_{2}-y\right|^{\alpha-n}\left(1+\lambda\left|x_{2}-y\right|\right) \mid d y \\
\leq & C \max \left(\left|x_{1}-x_{2}\right|^{\alpha-n},\left|x_{1}-x_{2}\right|\right) \int_{\Omega} f_{j}^{q}(y) f_{*}^{1-q}(y) d y \\
\leq & C \max \left(\left|x_{1}-x_{2}\right|^{\alpha-n},\left|x_{1}-x_{2}\right|\right)\left(\int_{\Omega} f_{j}(y) d y\right)^{q}\left(\int_{\Omega} f_{*}(y) d y\right)^{1-q} \\
\leq & C \max \left(\left|x_{1}-x_{2}\right|^{\alpha-n},\left|x_{1}-x_{2}\right|\right) .
\end{aligned}
$$

Thus $\int_{\Omega} f_{j}^{q}(y) f_{*}^{1-q}(y)|x-y|^{\alpha-n}(1+\lambda|x-y|) d y$ is equicontinuous in $\bar{\Omega}$. It follows that, as $j \rightarrow \infty$,

$$
\int_{\Omega} f_{j}^{q}(y) f_{*}^{1-q}(y)|x-y|^{\alpha-n}(1+\lambda|x-y|) d y \rightarrow \int_{\Omega} f_{*}(y)|x-y|^{\alpha-n}(1+\lambda|x-y|) d y
$$

uniformly for $x \in \bar{\Omega}$.

Therefore for any $\epsilon>0$ small enough, there exists $j_{0} \in \mathbb{N}$ such that for any $j>j_{0}$,

$$
\begin{aligned}
& \int_{\Omega} \int_{\Omega} f_{j}^{q}(x) f_{*}^{1-q}(x)|x-y|^{\alpha-n}(1+\lambda|x-y|) f_{j}^{q}(y) f_{*}^{1-q}(y) d x d y \\
\geq & \int_{\Omega} f_{j}^{q}(x) f_{*}^{1-q}(x)\left[\int_{\Omega}|x-y|^{\alpha-n}(1+\lambda|x-y|) f_{*}(y) d y-\epsilon\right] d x .
\end{aligned}
$$

By Hölder's inequality we know $\int_{\Omega} f_{j}^{q}(x) f_{*}^{1-q}(x) d x \leq C$. Again, notice that

$$
\begin{aligned}
& \int_{\Omega} f_{j}^{q}(x) f_{*}^{1-q}(x) \int_{\Omega}|x-y|^{\alpha-n}(1+\lambda|x-y|) f_{*}(y) d y d x \\
\rightarrow & \int_{\Omega} \int_{\Omega} f_{*}(x)|x-y|^{\alpha-n}(1+\lambda|x-y|) f_{*}(y) d y d x
\end{aligned}
$$

since $f_{*}^{1-q}(x) \int_{\Omega}|x-y|^{\alpha-n}(1+\lambda|x-y|) f_{*}(y) d y \in L^{\frac{1}{1-q}}(\Omega)$. We have for $j>j_{0}$ large enough,

$$
\begin{aligned}
& \int_{\Omega} \int_{\Omega} f_{j}^{q}(x) f_{*}^{1-q}(x)|x-y|^{\alpha-n}(1+\lambda|x-y|) f_{j}^{q}(y) f_{*}^{1-q}(y) d x d y \\
\geq & \int_{\Omega} \int_{\Omega} f_{*}(x)|x-y|^{\alpha-n}(1+\lambda|x-y|) f_{*}(y) d y d x-\epsilon-C \epsilon .
\end{aligned}
$$

Similarly, we also have, for $j>j_{0}$ large enough,

$$
\begin{aligned}
& \int_{\Omega} \int_{\Omega} f_{j}^{q}(x) f_{*}^{1-q}(x)|x-y|^{\alpha-n}(1+\lambda|x-y|) f_{j}^{q}(y) f_{*}^{1-q}(y) d x d y \\
\leq & \int_{\Omega} \int_{\Omega} f_{*}(x)|x-y|^{\alpha-n}(1+\lambda|x-y|) f_{*}(y) d y d x+\epsilon+C \epsilon .
\end{aligned}
$$


Therefore, as $j \rightarrow \infty$,

$$
\begin{aligned}
& \int_{\Omega} \int_{\Omega} f_{j}^{q}(x) f_{*}^{1-q}(x)|x-y|^{\alpha-n}(1+\lambda|x-y|) f_{j}^{q}(y) f_{*}^{1-q}(y) d x d y \\
\rightarrow & \int_{\Omega} \int_{\Omega} f_{*}(x)|x-y|^{\alpha-n}(1+\lambda|x-y|) f_{*}(y) d y d x .
\end{aligned}
$$

It follows from the above and Hölder's inequality that

$$
\begin{aligned}
& \liminf _{j \rightarrow \infty} \int_{\Omega} \int_{\Omega} f_{j}(x)|x-y|^{\alpha-n}(1+\lambda|x-y|) f_{j}(y) d x d y \\
\geq & \int_{\Omega} \int_{\Omega} f_{*}(x)|x-y|^{\alpha-n}(1+\lambda|x-y|) f_{*}(y) d x d y .
\end{aligned}
$$

Since $\left\|f_{j}\right\|_{L^{q}(\Omega)} \rightarrow\left\|f_{*}\right\|_{L^{q}(\Omega)}>0$, the above inequality then implies

$$
\begin{aligned}
& \liminf _{j \rightarrow \infty} \frac{\int_{\Omega} \int_{\Omega} f_{j}(x)|x-y|^{\alpha-n}(1+\lambda|x-y|) f_{j}(y) d x d y}{\left\|f_{j}\right\|_{L^{q}(\Omega)}^{2}} \\
\geq & \frac{\int_{\Omega} \int_{\Omega} f_{*}(x)|x-y|^{\alpha-n}(1+\lambda|x-y|) f_{*}(y) d x d y}{\left\|f_{*}\right\|_{L^{q}(\Omega)}^{2}} .
\end{aligned}
$$

That is: $f_{*}$ is a minimizer.

Now we are left to prove the claim: $\left\|f_{j}\right\|_{L^{1}(\Omega)} \leq C$.

From $\left\|f_{j}\right\|_{L^{q_{\alpha}(\Omega)}}=1$, we have

$$
\begin{aligned}
& \int_{\Omega} \int_{\Omega} f_{j}(x)|x-y|^{\alpha-n}(1+\lambda|x-y|) f_{j}(y) d x d y \\
\geq & (1-|\lambda| d(\Omega)) \int_{\Omega} \int_{\Omega} f_{j}(x)|x-y|^{\alpha-n} f_{j}(y) d x d y \\
\geq & (1-|\lambda| d(\Omega)) N_{\alpha}\left\|f_{j}\right\|_{L^{q_{\alpha}(\Omega)}}^{2} \\
\geq & C>0 .
\end{aligned}
$$

Since $\left\{f_{j}\right\}_{j=1}^{\infty}$ is a minimizing nonnegative sequence, we conclude that $\left\|f_{j}\right\|_{L^{q}(\Omega)} \geq$ $C_{1}(n, \alpha, q)>0$. By Hölder's inequality, we have

$$
0<C_{1}(n, \alpha, q) \leq\left\|f_{j}\right\|_{L^{q}(\Omega)} \leq C_{2}(n, \alpha, q)\left\|f_{j}\right\|_{L^{q_{\alpha}(\Omega)}}=C_{2}(n, \alpha, q) .
$$

The upper bound on $\left\|f_{j}\right\|_{L^{q}(\Omega)}$ indicates that

$$
\int_{\Omega} \int_{\Omega} f_{j}(x)|x-y|^{\alpha-n} f_{j}(y) d x d y<C_{3}(n, \alpha, q) .
$$

It follows, via reversed Hölder's inequality, that

$$
\left\|I_{\alpha, \Omega} f_{j}\right\|_{L^{q^{\prime}}(\Omega)} \leq C_{4}(n, \alpha, q)<\infty .
$$

Further, Hölder's inequality and reversed HLS inequality yield that

$$
\begin{aligned}
\infty>C_{4}(n, \alpha, q) & \geq\left\|I_{\alpha, \Omega} f_{j}\right\|_{L^{q^{\prime}}(\Omega)} \\
& \geq C_{5}(n, \alpha, q)\left\|I_{\alpha, \Omega} f_{j}\right\|_{L^{p_{\alpha}}(\Omega)} \\
& \geq C_{6}(n, \alpha, q)\left\|f_{j}\right\|_{L^{q_{\alpha}}(\Omega)}=C_{6}(n, \alpha, q)>0 .
\end{aligned}
$$

Then we can show that for $M>0$ such that $M^{q^{\prime}}|\Omega|<\frac{1}{2}\left(C_{4}(n, \alpha, q)\right)^{q^{\prime}}$, there exists $0<\delta<|\Omega|$ such that

$$
m\left\{x: I_{\alpha, \Omega} f_{j}(x) \leq M\right\}>\delta, \text { for all } j .
$$


In fact, for $\Omega_{1}:=\left\{x: I_{\alpha, \Omega} f_{j}(x) \leq M\right\}$,

$$
\begin{aligned}
& \left(C_{4}(n, \alpha, q)\right)^{q^{\prime}} \\
\leq & \int_{\Omega}\left(I_{\alpha, \Omega} f_{j}\right)^{q^{\prime}} d x=\int_{\Omega \backslash \Omega_{1}}\left(I_{\alpha, \Omega} f_{j}\right)^{q^{\prime}} d x+\int_{\Omega_{1}}\left(I_{\alpha, \Omega} f_{j}\right)^{q^{\prime}} d x \\
\leq & M^{q^{\prime}}|\Omega|+\left(\int_{\Omega_{1}}\left(I_{\alpha, \Omega} f_{i}\right)^{p_{\alpha}} d x\right)^{\frac{q^{\prime}}{p_{\alpha}}}\left|\Omega_{1}\right|^{1-\frac{q^{\prime}}{p_{\alpha}}} \\
\leq & M^{q^{\prime}}|\Omega|+\left(\int_{\Omega}\left(I_{\alpha, \Omega} f_{i}\right)^{p_{\alpha}} d x\right)^{\frac{q^{\prime}}{p_{\alpha}}}\left|\Omega_{1}\right|^{1-\frac{q^{\prime}}{p_{\alpha}}} \\
\leq & M^{q^{\prime}}|\Omega|+\left(\frac{C_{6}(n, \alpha, q)}{C_{5}(n, \alpha, q)}\right)^{q^{\prime}}\left|\Omega_{1}\right|^{1-\frac{q^{\prime}}{p_{\alpha}}} \\
< & \frac{1}{2}\left(C_{4}(n, \alpha, q)\right)^{q^{\prime}}+\left|\Omega_{1}\right|^{1-\frac{q^{\prime}}{p_{\alpha}}}\left(\frac{C_{6}(n, \alpha, q)}{C_{5}(n, \alpha, q)}\right)^{q^{\prime}},
\end{aligned}
$$

which yields the existence of such $\delta>0$.

Due to (3.3) we know that there exists $\epsilon_{0}>0$, such that for any $j$, we can find two points $x_{j}^{1}, x_{j}^{2} \in \Omega$ with the properties that $\left|x_{j}^{1}-x_{j}^{2}\right| \geq \epsilon_{0}$ and

$$
I_{\alpha, \Omega} f_{j}\left(x_{j}^{1}\right)=\int_{\Omega} f_{j}(y)\left|x_{j}^{1}-y\right|^{\alpha-n} \leq M, I_{\alpha, \Omega} f_{j}\left(x_{j}^{2}\right)=\int_{\Omega} f_{j}(y)\left|x_{j}^{2}-y\right|^{\alpha-n} \leq M .
$$

So

$$
\begin{aligned}
\int_{\Omega} f_{j}(y) d y \leq & \int_{\Omega \backslash B\left(x_{j}^{1}, \frac{\epsilon_{0}}{4}\right)} f_{j}(y) d y+\int_{\Omega \backslash B\left(x_{j}^{2}, \frac{\epsilon_{0}}{4}\right)} f_{j}(y) d y \\
\leq & \left(\frac{4}{\epsilon_{0}}\right)^{\alpha-n} \int_{\Omega \backslash B\left(x_{j}^{1}, \frac{\epsilon_{0}}{4}\right)} f_{j}(y)\left|x_{j}^{1}-y\right|^{\alpha-n} d y \\
& +\left(\frac{4}{\epsilon_{0}}\right)^{\alpha-n} \int_{\Omega \backslash B\left(x_{j}^{1}, \frac{\epsilon_{0}}{4}\right)} f_{j}(y)\left|x_{j}^{2}-y\right|^{\alpha-n} d y \\
\leq & \left(\frac{4}{\epsilon_{0}}\right)^{\alpha-n} 2 M,
\end{aligned}
$$

uniformly for all $j$. We thus verify the claim, and hereby, complete the proof of Lemma 3.2

It is standard to check that the minimizer $f(x)$ for energy $\xi_{\alpha, q}(\Omega)$ is positive, and, up to a constant multiplier, satisfies the following equation:

$$
f^{q-1}(x)=\int_{\Omega} \frac{f(y)}{|x-y|^{n-\alpha}} d y+\lambda \int_{\Omega} \frac{f(y)}{|x-y|^{n-\alpha-1}} d y, \quad x \in \bar{\Omega} .
$$

From the proof of Lemma 3.2, we also know that $f(x) \in L^{1}(\Omega)$, thus $f^{q-1}(x) \in$ $L^{\infty}(\Omega)$ by equation (3.4).

Writing $u(x)=f^{q-1}(x), p=q^{\prime}$, we thus find a weak positive solution $u(x) \in$ $L^{p}(\Omega)$ to

$$
u(x)=\int_{\Omega} \frac{u^{p-1}(y)}{|x-y|^{n-\alpha}} d y+\lambda \int_{\Omega} \frac{u^{p-1}(y)}{|x-y|^{n-\alpha-1}} d y, \quad x \in \bar{\Omega}
$$

for $0>p>\frac{2 n}{n-\alpha}=p_{\alpha}$. To complete the proof of part (1) in Theorem 1.1, we need to show that $u \in C^{1}(\bar{\Omega})$. 
In fact, $f(x) \in L^{1}(\Omega)$ implies

$$
\int_{\Omega} u^{p-1}(y) d y<\infty
$$

It is easy to see that $u \in C(\bar{\Omega})$ from equation (3.5). To show $u \in C^{1}(\bar{\Omega})$, we can directly compute, for $i=1, \ldots, n$,

$$
\partial_{x_{i}} u(x)=-(n-\alpha) \int_{\Omega} \frac{u^{p-1}(y)\left(x_{i}-y_{i}\right)}{|x-y|^{n-\alpha+2}} d y \in C(\bar{\Omega}) .
$$

Part (1) of Theorem 1.1 is hereby proved.

It is interesting to study some properties about the positive solutions to the new integral equation (3.5) (such as multiplicity of solutions, blowup behavior as $q \rightarrow q_{\alpha}$ in a star-shaped domain, etc.) In the rest of this section, as in [5, we will show that even though the boundary condition is not given pointwise, the symmetric property for solutions to the integral equation (3.5) with $\lambda=0$ on a unit ball still holds. Contrary to the result in [5], here we will show that the solution is monotone increasing due to the monotone increasing property of the kernel.

On $B_{1}:=B_{1}(0)=\left\{x \in \mathbb{R}^{n}|| x \mid<1, x \in \mathbb{R}^{n}\right\}$, for $\lambda=0$ we rewrite the equation (3.5) as

We have

$$
u(x)=\int_{B_{1}} \frac{u^{p-1}(y)}{|x-y|^{n-\alpha}} d y, \quad x \in B_{1}(0) .
$$

Theorem 3.3. Let $\alpha>n, p \in\left(p_{\alpha}, 0\right)$. Then every positive solution $u \in L^{p}\left(\overline{B_{1}}\right)$ to (3.6) is radially symmetric about the origin and strictly increasing in the radial direction.

Easy to see from the proof of Part (1) of Theorem 1.1 that $u \in C^{1}\left(\overline{B_{1}}\right)$. We will use the method of moving planes to prove Theorem 3.3

Firstly, we recall the idea of the method of moving planes in $B_{1}$ (see e.g. [3, 5]).

For any real number $\lambda \in(-1,0)$, define $T_{\lambda}=\left\{x \in \mathbb{R}^{n} \mid x_{1}=\lambda\right\}$, and $x^{\lambda}=$ $\left(2 \lambda-x_{1}, x_{2}, \cdots, x_{n}\right)$ as the reflection of point $x=\left(x_{1}, x_{2}, \cdots, x_{n}\right)$ about plane $T_{\lambda}$. Let

$$
\Sigma_{\lambda}=\left\{x=\left(x_{1}, x_{2}, \cdots, x_{n}\right) \in B_{1} \mid-1<x_{1}<\lambda\right\},
$$

and $\Sigma_{\lambda}^{C}=B_{1} \backslash \bar{\Sigma}_{\lambda}$ be the complement of $\Sigma_{\lambda}$ in $B_{1}$. Set $u_{\lambda}(x)=u\left(x^{\lambda}\right)$. We shall complete the proof in two steps. In step 1 , we show that for $\lambda$ sufficiently close to $-1$

$$
u(x) \geq u_{\lambda}(x), \quad \forall x \in \Sigma_{\lambda} .
$$

Then we can start to move plane $T_{\lambda}$ along the $x_{1}$ direction. In step 2 , we move the plane to the right as long as inequality (3.7) holds. We show that the plane can be moved to $\lambda=0$. So

$$
u\left(-x_{1}, x_{2}, \cdots, x_{n}\right) \geq u\left(x_{1}, x_{2}, \cdots, x_{n}\right), \quad \forall x \in B_{1}, x_{1} \geq 0 .
$$

Similarly, we can start to move plane $T_{\lambda}$ from a place close to $\lambda=1$, and move it to the left limiting position $T_{0}$. Then

$$
u\left(-x_{1}, x_{2}, \cdots, x_{n}\right) \leq u\left(x_{1}, x_{2}, \cdots, x_{n}\right), \quad \forall x \in B_{1}, x_{1} \geq 0 .
$$

By (3.8) and (3.9), we have that $u(x)$ is symmetric about the plane $x_{1}=0$. Similarly, we can show that $u(x)$ is symmetric about any plane passing through the 
origin, which then implies that $u(x)$ is radially symmetric about the origin and strictly increasing in the radial direction.

First, we have following comparison inequality.

Lemma 3.4. For any $x \in \Sigma_{\lambda}$ with $\lambda \in(-1,0)$, it holds

$$
u(x)-u_{\lambda}(x) \geq \int_{\Sigma_{\lambda}}\left[\frac{1}{|x-y|^{n-\alpha}}-\frac{1}{\left|x^{\lambda}-y\right|^{n-\alpha}}\right]\left(u^{p-1}(y)-u_{\lambda}^{p-1}(y)\right) d y .
$$

Proof. Let $\widetilde{\Sigma}_{\lambda}=\left\{x^{\lambda} \mid x \in \Sigma_{\lambda}\right\}$ be the reflection of $\Sigma_{\lambda}$ about plane $T_{\lambda}$, then

$$
\begin{aligned}
u(x) & =\int_{\Sigma_{\lambda}} \frac{u^{p-1}(y)}{|x-y|^{n-\alpha}} d y+\int_{\widetilde{\Sigma}_{\lambda}} \frac{u^{p-1}(y)}{|x-y|^{n-\alpha}} d y \\
& +\int_{\Sigma_{\lambda}^{C} \backslash \widetilde{\Sigma}_{\lambda}} \frac{u^{p-1}(y)}{|x-y|^{n-\alpha}} d y .
\end{aligned}
$$

Noting that $|x-y|>\left|x^{\lambda}-y\right|$ in $x \in \Sigma_{\lambda}, y \in \Sigma_{\lambda}^{C} \backslash \widetilde{\Sigma}_{\lambda}$, we have

$$
\begin{aligned}
u(x)-u_{\lambda}(x)= & \int_{\Sigma_{\lambda}}\left[\frac{1}{|x-y|^{n-\alpha}}-\frac{1}{\left|x^{\lambda}-y\right|^{n-\alpha}}\right] u^{p-1}(y) d y \\
& +\int_{\widetilde{\Sigma}_{\lambda}}\left[\frac{1}{|x-y|^{n-\alpha}}-\frac{1}{\left|x^{\lambda}-y\right|^{n-\alpha}}\right] u^{p-1}(y) d y \\
& +\int_{\Sigma_{\lambda}^{C} \backslash \widetilde{\Sigma}_{\lambda}}\left[\frac{1}{|x-y|^{n-\alpha}}-\frac{1}{\left|x^{\lambda}-y\right|^{n-\alpha}}\right] u^{p-1}(y) d y \\
\geq & \int_{\Sigma_{\lambda}}\left[\frac{1}{|x-y|^{n-\alpha}}-\frac{1}{\left|x^{\lambda}-y\right|^{n-\alpha}}\right] u^{p-1}(y) d y \\
& +\int_{\Sigma_{\lambda}}\left[\frac{1}{\left|x-y^{\lambda}\right|^{n-\alpha}}-\frac{1}{\left|x^{\lambda}-y^{\lambda}\right|^{n-\alpha}}\right] u_{\lambda}^{p-1}(y) d y \\
= & \int_{\Sigma_{\lambda}}\left[\frac{1}{|x-y|^{n-\alpha}}-\frac{1}{\left|x^{\lambda}-y\right|^{n-\alpha}}\right] u^{p-1}(y) d y \\
& -\int_{\Sigma_{\lambda}}\left[\frac{1}{|x-y|^{n-\alpha}}-\frac{1}{\left|x^{\lambda}-y\right|^{n-\alpha}}\right] u_{\lambda}^{p-1}(y) d y \\
= & \int_{\Sigma_{\lambda}}\left[\frac{1}{|x-y|^{n-\alpha}}-\frac{1}{\left|x^{\lambda}-y\right|^{n-\alpha}}\right]\left(u^{p-1}(y)-u_{\lambda}^{p-1}(y)\right) d y .
\end{aligned}
$$

\section{Proof of Theorem 3.3 .}

Step 1. Let $u \in C^{1}\left(\overline{B_{1}}\right)$ be a positive solution to equation (3.6). We show that for $\lambda$ sufficiently close to -1 , inequality (3.7) holds.

From (3.6) we have

$$
\begin{aligned}
\left.\frac{\partial u(x)}{\partial x_{1}}\right|_{x_{1}=-1} & =(\alpha-n) \int_{B_{1}}|x-y|^{\alpha-n-2}\left(-1-y_{1}\right) u^{p-1}(y) d y \\
& <0 .
\end{aligned}
$$

Therefore for $\lambda$ sufficiently close to -1 , we have

$$
u(x) \geq u_{\lambda}(x) \text { for } x \in \Sigma_{\lambda} .
$$

Step 2. Plane $T_{\lambda}$ can be moved continuously towards right to its limiting position as long as inequality (3.7) holds. 
Define

$$
\lambda_{0}=\sup \left\{\lambda \in[-1,0) \mid u(y) \geq u_{\mu}(y), \forall y \in \Sigma_{\mu}, \mu \leq \lambda\right\} .
$$

We claim that $\lambda_{0}$ must be 0 .

We prove it by contradiction. Suppose not, that is, $\lambda_{0}<0$.

We first show that

$$
u(x)>u_{\lambda_{0}}(x), \quad \text { in } \Sigma_{\lambda_{0}}
$$

Hence, we have

$$
u(x)-u_{\lambda_{0}}(x)>c_{1}>0, \quad \text { in } \Sigma_{\lambda_{0}-\epsilon_{1}}
$$

for $\epsilon_{1}>0$ small enough.

In fact, since $|x-y|<\left|x-y^{\lambda_{0}}\right|$ for $x, y \in \Sigma_{\lambda_{0}}$, we have, similar to the calculation in the proof of Lemma 3.4, that

$$
\begin{aligned}
u(x)-u_{\lambda_{0}}(x) & =\int_{\Sigma_{\lambda_{0}}}\left[\frac{1}{|x-y|^{n-\alpha}}-\frac{1}{\left|x^{\lambda_{0}}-y\right|^{n-\alpha}}\right]\left(u^{p-1}(y)-u_{\lambda_{0}}^{p-1}(y)\right) d y \\
& +\int_{\Sigma_{\lambda_{0}}^{C} \backslash \widetilde{\Sigma}_{\lambda_{0}}}\left[\frac{1}{|x-y|^{n-\alpha}}-\frac{1}{\left|x^{\lambda_{0}}-y\right|^{n-\alpha}}\right] u^{p-1}(y) d y \\
& \geq \int_{\Sigma_{\lambda_{0}}^{C} \backslash \widetilde{\Sigma}_{\lambda_{0}}}\left[\frac{1}{|x-y|^{n-\alpha}}-\frac{1}{\left|x^{\lambda_{0}}-y\right|^{n-\alpha}}\right] u^{p-1}(y) d y
\end{aligned}
$$

If there exists some point $x_{0} \in \Sigma_{\lambda_{0}}$ such that $u\left(x_{0}\right)=u_{\lambda_{0}}\left(x_{0}\right)$, then since $|x-y|>$ $\left|x^{\lambda_{0}}-y\right|$ for $x \in \Sigma_{\lambda_{0}}, y \in \Sigma_{\lambda_{0}}^{C}$, we deduce from (3.11) that

$$
u(y) \equiv \infty, \quad \forall y \in \Sigma_{\lambda_{0}}^{C} \backslash \widetilde{\Sigma}_{\lambda_{0}} .
$$

This contradicts to the assumption that $u \in C^{1}\left(\overline{B_{1}}\right)$ is a positive solution.

For some small $\delta_{1}>0$, we choose $\varepsilon \in\left(0, \epsilon_{1}\right)$ small enough such that for any $\lambda \in\left[\lambda_{0}, \lambda_{0}+\varepsilon\right)$, there holds

$$
u(x) \geq u_{\lambda}(x), \forall x \in \Sigma_{\lambda_{0}-\varepsilon_{1}}
$$

and

$$
\left|\frac{1}{|x-y|^{n-\alpha}}-\frac{1}{\left|x^{\lambda}-y\right|^{n-\alpha}}\right| \leq \delta_{1} \quad \text { for } x \in \Sigma_{\lambda} \backslash \Sigma_{\lambda_{0}-\varepsilon_{1}} .
$$

Write

$$
\Sigma_{\lambda}^{u}=\left\{x \in \Sigma_{\lambda} \mid u_{\lambda}(x)>u(x)\right\} .
$$

It follows from (3.10) that for any $x \in \Sigma_{\lambda}^{u}$,

$$
\begin{aligned}
0>u(x)-u_{\lambda}(x) & \geq \int_{\Sigma_{\lambda}}\left[\frac{1}{|x-y|^{n-\alpha}}-\frac{1}{\left|x^{\lambda}-y\right|^{n-\alpha}}\right]\left(u^{p-1}(y)-u_{\lambda}^{p-1}(y)\right) d y \\
& \geq \int_{\Sigma_{\lambda}^{u}}\left[\frac{1}{|x-y|^{n-\alpha}}-\frac{1}{\left|x^{\lambda}-y\right|^{n-\alpha}}\right]\left(u^{p-1}(y)-u_{\lambda}^{p-1}(y)\right) d y \\
& \geq-\delta_{1} \int_{\Sigma_{\lambda}^{u}}\left(u^{p-1}(y)-u_{\lambda}^{p-1}(y)\right) d y .
\end{aligned}
$$


Since $u \in C^{1}\left(\overline{B_{1}}\right)$, there exists a positive constant $C_{0}$ such that $\frac{1}{C_{0}} \leq u \leq C_{0}$. It follows from the above

$$
\begin{aligned}
\int_{\Sigma_{\lambda}^{u}}\left(u_{\lambda}(x)-u(x)\right) d x & \leq \delta_{1} \int_{\Sigma_{\lambda}^{u}} \int_{\Sigma_{\lambda}^{u}}\left(u^{p-1}(y)-u_{\lambda}^{p-1}(y)\right) d y d x \\
& \leq(1-p) \delta_{1} \int_{\Sigma_{\lambda}^{u}} \int_{\Sigma_{\lambda}^{u}} u^{p-2}(y)\left(u_{\lambda}(y)-u(y)\right) d y d x \\
& \leq C \delta_{1}\left(\varepsilon+\varepsilon_{1}\right)^{n} \int_{\Sigma_{\lambda}^{u}}\left(u_{\lambda}(y)-u(y)\right) d y .
\end{aligned}
$$

It implies that

$$
\left\|u_{\lambda}-u\right\|_{L^{1}\left(\Sigma_{\lambda}^{u}\right)} \equiv 0
$$

for $\delta_{1}, \varepsilon, \varepsilon_{1}$ small enough, and hence $\Sigma_{\lambda}^{u}$ must have measure zero.

We thus have

$$
u(x)-u_{\lambda}(x) \geq 0, \text { for any } x \in \Sigma_{\lambda}, \forall \lambda \in\left[\lambda_{0}, \lambda_{0}+\varepsilon\right)
$$

since $u$ is continuous. This contradicts to the definition of $\lambda_{0}$. Hence, $\lambda_{0}=0$. We hereby complete the proof of Theorem 3.4.

\section{Existence RESUlt For CRitical CASE}

In this section, we study the existence of positive solutions to the integral equation with critical exponent.

The non-existence of positive solution to (1.1) with critical exponent for $\lambda \geq 0$ on a start-shaped domain follows from Pohozaev identity (2.3). Next, we shall establish the existence as well as the regularity results for weak solutions to (1.1) with critical exponent for $\lambda<0$. To this end, we consider

$$
Q_{\lambda}(\Omega):=\inf _{f \in L_{+}^{q_{\alpha}}(\Omega)} \frac{\int_{\Omega} \int_{\Omega} f(x)\left(|x-y|^{-(n-\alpha)}+\lambda|x-y|^{-(n-\alpha-1)}\right) f(y) d y d x}{\|f\|_{L^{q_{\alpha}(\Omega)}}^{2}} .
$$

Notice that the corresponding Euler-Lagrange equation for extremal functions, up to a constant multiplier, is integral equation (1.1) with $q=q_{\alpha}$.

First, we show

Lemma 4.1. $Q_{\lambda}(\Omega)<N_{\alpha}$ for all $\lambda<0$. Further, $0<Q_{\lambda}(\Omega)<N_{\alpha}$ for any $\lambda \in\left(-\frac{1}{d(\Omega)}, 0\right)$.

Proof. Let $x_{*} \in \Omega$. For small positive $\epsilon$ and a fixed $R>0$ so that $B_{R}\left(x_{*}\right) \subset \Omega$, we define

$$
\tilde{f}_{\epsilon}(x)= \begin{cases}f_{\epsilon}(x) & x \in B_{R}\left(x_{*}\right) \subset \Omega, \\ 0 & x \in \mathbb{R}^{n} \backslash B_{R}\left(x_{*}\right),\end{cases}
$$


where $f_{\epsilon}$ is given by (2.1). Obviously, $\tilde{f}_{\epsilon} \in L^{q_{\alpha}}\left(\mathbb{R}^{n}\right)$. Thus, similar to the proof of Proposition 2.1, we have

$$
\begin{aligned}
& \int_{\Omega} \int_{\Omega}\left(\frac{1}{|x-y|^{n-\alpha}}+\frac{\lambda}{|x-y|^{n-\alpha-1}}\right) \tilde{f}_{\epsilon}(x) \tilde{f}_{\epsilon}(y) d x d y \\
& =\int_{\mathbb{R}^{n}} \int_{\mathbb{R}^{n}} \frac{1}{|x-y|^{n-\alpha}} f_{\epsilon}(x) f_{\epsilon}(y) d x d y \\
& -2 \int_{\mathbb{R}^{n}} \int_{\mathbb{R}^{n} \backslash B_{R}\left(x_{*}\right)} \frac{f_{\epsilon}(x) f_{\epsilon}(y)}{|x-y|^{n-\alpha}} d x d y+\int_{\mathbb{R}^{n} \backslash B_{R}\left(x_{*}\right)} \int_{\mathbb{R}^{n} \backslash B_{R}\left(x_{*}\right)} \frac{f_{\epsilon}(x) f_{\epsilon}(y)}{|x-y|^{n-\alpha}} d x d y \\
& +\lambda \int_{B_{R}\left(x_{*}\right)} \int_{B_{R}\left(x_{*}\right)} \frac{f_{\epsilon}(x) f_{\epsilon}(y)}{|x-y|^{n-\alpha-1}} d x d y \\
& \leq N_{\alpha}\left\|f_{\epsilon}\right\|_{L^{q_{\alpha}\left(\mathbb{R}^{n}\right)}}^{2}-C_{1}\left(\frac{\epsilon}{R}\right)^{n}+\lambda J_{1},
\end{aligned}
$$

where

$$
\begin{aligned}
J_{1} & :=\int_{B_{R}\left(x_{*}\right)} \int_{B_{R}\left(x_{*}\right)} \frac{f_{\epsilon}(x) f_{\epsilon}(y)}{|x-y|^{n-\alpha-1}} d x d y \\
& =\int_{B_{R}\left(x_{*}\right)} \int_{B_{R}\left(x_{*}\right)}|x-y|^{-(n-\alpha-1)}\left(\frac{\epsilon}{\epsilon^{2}+\left|x-x_{*}\right|^{2}}\right)^{\frac{n+\alpha}{2}}\left(\frac{\epsilon}{\epsilon^{2}+\left|y-x_{*}\right|^{2}}\right)^{\frac{n+\alpha}{2}} d x d y \\
& =\epsilon^{-(n-\alpha-1)-(n+\alpha)} \int_{B_{R}(0)} \int_{B_{R}(0)}\left|\frac{x-y}{\epsilon}\right|^{-(n-\alpha-1)}\left(1+\left|\frac{x}{\epsilon}\right|^{2}\right)^{-\frac{n+\alpha}{2}}\left(1+\left|\frac{y}{\epsilon}\right|^{2}\right)^{-\frac{n+\alpha}{2}} d x d y \\
& =\epsilon \int_{B_{\frac{R}{\epsilon}}(0)} \int_{B_{\frac{R}{\epsilon}}(0)}|\xi-\eta|^{-(n-\alpha-1)}\left(1+|\xi|^{2}\right)^{-\frac{n+\alpha}{2}}\left(1+|\eta|^{2}\right)^{-\frac{n+\alpha}{2}} d \xi d \eta \\
& \geq C_{0} \epsilon .
\end{aligned}
$$

So, for $\lambda<0$ and small enough $\epsilon>0$, we have

$$
\begin{aligned}
& \int_{\Omega} \int_{\Omega}\left(\frac{1}{|x-y|^{n-\alpha}}+\frac{\lambda}{|x-y|^{n-\alpha-1}}\right) \tilde{f}_{\epsilon}(x) \tilde{f}_{\epsilon}(y) d x d y \\
\leq & N_{\alpha}\left\|f_{\epsilon}\right\|_{L^{q_{\alpha}\left(\mathbb{R}^{n}\right)}}^{2}-C_{1}\left(\frac{\epsilon}{R}\right)^{n}+\lambda C_{0} \epsilon .
\end{aligned}
$$

This implies that $Q_{\lambda}(\Omega)<N_{\alpha}$ for all $\lambda<0$.

On the other hand, it is easy to see that $Q_{\lambda}(\Omega)>0$ for any $\lambda \in\left(-\frac{1}{d(\Omega)}, 0\right)$.

The existence of solutions to equation (1.1) will follow from the existence of a minimizer for energy $Q_{\lambda}(\Omega)$.

Proposition 4.2. For any $\lambda \in\left(-\frac{1}{d(\Omega)}, 0\right)$, infimum $Q_{\lambda}(\Omega)$ is achieved by a positive function $f_{*} \in L^{q_{\alpha}}(\Omega)$.

For $q<q_{\alpha}$, consider

$Q_{\lambda, q}(\Omega)=\inf _{f \in L_{+}^{q}(\Omega)} \frac{\int_{\Omega} \int_{\Omega} f(x)|x-y|^{-(n-\alpha)} f(y) d x d y+\lambda \int_{\Omega} \int_{\Omega} f(x)|x-y|^{-(n-\alpha-1)} f(y) d x d y}{\|f\|_{L^{q}(\Omega)}^{2}}$.

By Lemma 3.2, the infimum is attained by a positive function $f_{q}$, which satisfies the subcritical equation

$$
Q_{\lambda, q}(\Omega) f^{q-1}(x)=\int_{\Omega} \frac{f(y)}{|x-y|^{n-\alpha}} d y+\lambda \int_{\Omega} \frac{f(y)}{|x-y|^{n-\alpha-1}} d y, \quad x \in \bar{\Omega},
$$

and $\left\|f_{q}\right\|_{L^{q}(\Omega)}=1$. Further, we can show easily that $f_{q} \in C(\bar{\Omega})$ and $Q_{\lambda, q} \rightarrow Q_{\lambda}$ for $q \rightarrow\left(q_{\alpha}\right)^{-}$. 
Lemma 4.3. For $\lambda \in\left(-\frac{1}{d(\Omega)}, 0\right)$ and $q \in\left(0, q_{\alpha}\right)$, let $f_{q}>0$ be a minimal energy solution to (4.1) with $\left\|f_{q}\right\|_{L^{q}(\Omega)}=1$. If $0<Q_{\lambda, q} \leq N_{\alpha}-\epsilon$ for some $\epsilon>0$, then there exists $C>0$ such that $\frac{1}{C} \leq f_{q}(x) \leq C$ uniformly for all $x \in \bar{\Omega}$ and $q \in\left(0, q_{\alpha}\right)$.

Proof. It is easy to see that $\max _{\bar{\Omega}} f_{q}(x):=f_{q}\left(x_{q}\right) \leq C<\infty$ uniformly for all $x \in \bar{\Omega}$ and $q \in\left(0, q_{1}\right)$ provided $0<q_{1}<q_{\alpha}$.

We first prove by contradiction that $\max _{\bar{\Omega}} f_{q}(x)=f_{q}\left(x_{q}\right) \leq C<\infty$ uniformly for all $x \in \bar{\Omega}$ and $q \in\left(0, q_{\alpha}\right)$. Suppose not. Then $f_{q}\left(x_{q}\right) \rightarrow+\infty$ for $q \rightarrow\left(q_{\alpha}\right)^{-}$. Let

$$
\mu_{q}=f_{q}^{-\frac{2-q}{\alpha}}\left(x_{q}\right), \text { and } \Omega_{\mu}=\frac{\Omega-x_{q}}{\mu_{q}}:=\left\{z \mid z=\frac{x-x_{q}}{\mu_{q}} \text { for } x \in \Omega\right\} .
$$

Define

$$
g_{q}(z)=\mu_{q}^{\frac{\alpha}{2-q}} f_{q}\left(\mu_{q} z+x_{q}\right), \quad \text { for } z \in \bar{\Omega}_{\mu}
$$

Then $g_{q}$ satisfies

$$
Q_{\lambda, q}(\Omega) g_{q}^{q-1}(z)=\int_{\Omega_{\mu}} \frac{g_{q}(y)}{|z-y|^{n-\alpha}} d y+\lambda \mu_{q} \int_{\Omega_{\mu}} \frac{g_{q}(y)}{|z-y|^{n-\alpha-1}} d y, \quad z \in \bar{\Omega}_{\mu},
$$

and $g_{q}(0)=1, g_{q}(z) \in(0,1]$.

For convenience, denote $h_{q}(z):=g_{q}^{q-1}(z)$. Then (4.3) is equivalent to

$$
Q_{\lambda, q}(\Omega) h_{q}(z)=\int_{\Omega_{\mu}} \frac{h_{q}^{p-1}(y)}{|z-y|^{n-\alpha}} d y+\lambda \mu_{q} \int_{\Omega_{\mu}} \frac{h_{q}^{p-1}(y)}{|z-y|^{n-\alpha-1}} d y, \quad z \in \bar{\Omega}_{\mu},
$$

where $\frac{1}{p}+\frac{1}{q}=1, h_{q}(0)=1, h_{q}(z) \geq 1$.

Claim: There exist $C_{1}, C_{2}>0$ such that, for all $z$ in a domain $\widehat{\Omega}$ covered by $\Omega_{\mu}$, when $q \rightarrow\left(q_{\alpha}\right)^{-}$

$$
0<C_{1}\left(1+|z|^{\alpha-n}\right) \leq h_{q}(z) \leq C_{2}\left(1+|z|^{\alpha-n}\right), \text { uniformly. }
$$

We relegate the proof of this claim to the end.

Once the claim is proved, we can prove that $h_{q}(z)$ is equicontinuous on any bounded domain $\widehat{\Omega} \subset \Omega_{\mu}$ when $q \rightarrow\left(q_{\alpha}\right)^{-}$. We write

$$
\begin{aligned}
& Q_{\lambda, q}(\Omega) h_{q}(z) \\
= & \int_{\Omega_{\mu} \backslash B(0, R)} \frac{h_{q}^{p-1}(y)}{|z-y|^{n-\alpha}} d y+\int_{\Omega_{\mu} \cap B(0, R)} \frac{h_{q}^{p-1}(y)}{|z-y|^{n-\alpha}} d y \\
+ & \lambda \mu_{q} \int_{\Omega_{\mu} \backslash B(0, R)} \frac{h_{q}^{p-1}(y)}{|z-y|^{n-\alpha-1}} d y+\lambda \mu_{q} \int_{\Omega_{\mu} \cap B(0, R)} \frac{h_{q}^{p-1}(y)}{|z-y|^{n-\alpha-1}} d y .
\end{aligned}
$$

Notice that

$$
\int_{\Omega_{\mu} \backslash B(0, R)} \frac{h_{q}^{p-1}(y)}{|z-y|^{n-\alpha}}\left(1+\lambda \mu_{q}|z-y|\right) d y \geq(1-|\lambda| d(\Omega)) \int_{\Omega_{\mu} \backslash B(0, R)} \frac{h_{q}^{p-1}(y)}{|z-y|^{n-\alpha}} d y \geq 0 .
$$


Then for $\epsilon>0$ small enough, we have, by (4.5), that

$$
\begin{aligned}
0 & \leq \int_{\Omega_{\mu} \backslash B(0, R)} \frac{h_{q}^{p-1}(y)}{|z-y|^{n-\alpha}}\left(1+\lambda \mu_{q}|z-y|\right) d y \\
& \leq C \int_{\Omega_{\mu} \backslash B(0, R)} \frac{h_{q}^{p-1}(y)}{|y|^{n-\alpha}} d y \\
& \leq C \int_{R}^{\infty} r^{(\alpha-n)(p-1)+\alpha-1} d r \\
& =C R^{(\alpha-n)(p-1)+\alpha}<\epsilon
\end{aligned}
$$

for any $z \in \widehat{\Omega}$, by taking $R>0$ large enough and $q$ close to $q_{\alpha}$. Similarly, we have

$$
\left|\lambda \mu_{q} \int_{\Omega_{\mu} \cap B(0, R)} \frac{h_{q}^{p-1}(y)}{|z-y|^{n-\alpha-1}} d y\right|<\epsilon
$$

by taking $R>0$ large enough and $q$ close to $q_{\alpha}$. On the other hand, it is easy to see that $\int_{\Omega_{\mu} \cap B(0, R)} \frac{h_{q}^{p-1}(y)}{|z-y|^{n-\alpha}} d y \in C^{1}(\widehat{\Omega})$. Hence for $z_{1}, z_{2} \in \widehat{\Omega}$,

$$
\begin{aligned}
& \left|\int_{\Omega_{\mu} \cap B(0, R)} \frac{h_{q}^{p-1}(y)}{\left|z_{1}-y\right|^{n-\alpha}} d y-\int_{\Omega_{\mu} \cap B(0, R)} \frac{h_{q}^{p-1}(y)}{\left|z_{2}-y\right|^{n-\alpha}} d y\right| \\
\leq & \int_{\Omega_{\mu} \cap B(0, R)} h_{q}^{p-1}(y) \frac{1}{|\xi-y|^{n-\alpha+1}} d y\left|z_{1}-z_{2}\right| \\
\leq & \int_{B(0, R)} \frac{1}{|\xi-y|^{n-\alpha+1}} d y\left|z_{1}-z_{2}\right| \leq C R^{\alpha-1}\left|z_{1}-z_{2}\right|,
\end{aligned}
$$

where $\xi=t z_{1}+(1-t) z_{2}$ for some $t \in(0,1)$. By (4.6), (4.7) and (4.8) we conclude that $h_{q}(z)$ is equicontinuous on bounded domain $\widehat{\Omega} \in \mathbb{R}^{n}$ when $q \rightarrow\left(q_{\alpha}\right)^{-}$.

As $q \rightarrow\left(q_{\alpha}\right)^{-}$, there are two cases:

Case 1. $\Omega_{\mu} \rightarrow \mathbb{R}_{T}^{n}:=\left\{\left(z_{1}, z_{2}, \cdots, z_{n}\right) \mid z_{n}>T \geq 0\right\}$, and $h_{q}(z) \rightarrow h(z) \in C\left(\mathbb{R}_{T}^{n}\right)$ uniformly in any compact set in $\mathbb{R}_{T}^{n}$, where $h(z)$ satisfies

$$
Q_{\lambda} h(z)=\int_{\mathbb{R}_{T}^{n}} \frac{h^{p_{\alpha}-1}(y)}{|z-y|^{n-\alpha}} d y, \quad h(0)=1 .
$$

Also, direct computation yields

$$
1=\int_{\Omega} f_{q}^{q}(y) d y=\mu_{q}^{\left(\frac{n+\alpha}{2-q}\right) \cdot\left(\frac{2 n}{n+\alpha}-q\right)} \cdot \int_{\Omega_{\mu}} g_{q}^{q} d z \leq \int_{\Omega_{\mu}} g_{q}^{q} d z=\int_{\Omega_{\mu}} h_{q}^{p} d z
$$

On the other hand, by (4.5) we have $\int_{\Omega_{\mu}} h_{q}^{p} d z \leq C$ uniformly. Again by (4.5), $\int_{\mathbb{R}_{T}^{n}} h^{p_{\alpha}} d z=\lim _{q \rightarrow\left(q_{\alpha}\right)^{-}} \int_{\Omega_{\mu}} h_{q}^{p} d z \geq 1$. Denote $g(x)=h^{p_{\alpha}-1}(x)$. Then $\int_{\mathbb{R}_{T}^{n}} h^{p_{\alpha}} d z=$ 
$\int_{\mathbb{R}_{T}^{n}} g^{q_{\alpha}} d z$. By (4.9), we have

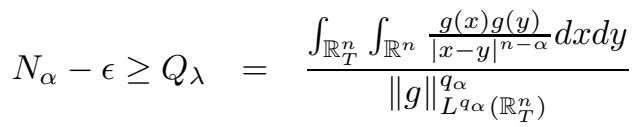

$$
\begin{aligned}
& \geq \frac{\int_{\mathbb{R}_{T}^{n}} \int_{\mathbb{R}_{T}^{n}} \frac{g(x) g(y)}{|x-y|^{n-\alpha}} d x d y}{\|g\|_{L^{q \alpha}\left(\mathbb{R}_{T}^{n}\right)}^{2}} \\
& =\frac{\int_{\mathbb{R}^{n}} \int_{\mathbb{R}^{n}} \frac{\tilde{g}(x) \tilde{g}(y)}{|x-y|^{n-\alpha}} d x d y}{\|\tilde{g}\|_{L^{q_{\alpha}\left(\mathbb{R}^{n}\right)}}^{2}} \geq N_{\alpha} .
\end{aligned}
$$

Contradiction!

Case 2. $\Omega_{\mu} \rightarrow \mathbb{R}^{n}$, and $h_{q}(z) \rightarrow h(z) \in C\left(\mathbb{R}^{n}\right)$ uniformly in any compact set in $\mathbb{R}^{n}$, where $h(z)$ satisfies

$$
Q_{\lambda} h(z)=\int_{\mathbb{R}^{n}} \frac{h^{p_{\alpha}-1}(y)}{|z-y|^{n-\alpha}} d y, \quad h(0)=1 .
$$

Similarly, $C \geq \int_{\mathbb{R}^{n}} h^{p_{\alpha}} d z \geq 1$. Denote $g(x)=h^{p_{\alpha}-1}(x)$. Then $\int_{\mathbb{R}^{n}} h^{p_{\alpha}} d z=$ $\int_{\mathbb{R}^{n}} g^{q_{\alpha}} d z$. By (4.10) we have

$$
N_{\alpha}-\epsilon \geq Q_{\lambda}=\frac{\int_{\mathbb{R}^{n}} \int_{\mathbb{R}^{n}} \frac{g(x) g(y)}{|x-y|^{n-\alpha}} d x d y}{\|g\|_{L^{q_{\alpha}}\left(\mathbb{R}^{n}\right)}^{q^{n}}} \geq \frac{\int_{\mathbb{R}^{n}} \int_{\mathbb{R}^{n}} \frac{g(x) g(y)}{|x-y|^{n-\alpha}} d x d y}{\|g\|_{L^{q_{\alpha}}\left(\mathbb{R}^{n}\right)}^{2}} \geq N_{\alpha},
$$

which again implies a contradiction.

Thus we conclude that there exists $C>0$ such that $f_{q}(y) \leq C$ uniformly in $y \in \bar{\Omega}$ and $q \in\left(0, q_{\alpha}\right)$.

On the other hand, if $\min _{\bar{\Omega}} f_{q}(x):=f_{q}\left(\tilde{x}_{q}\right) \rightarrow 0$ as $q \rightarrow\left(q_{\alpha}\right)^{-}$, by using $f_{q}(y) \leq C$ uniformly in $y \in \bar{\Omega}$ and $q \in\left(0, q_{\alpha}\right)$ we have

$$
\infty \leftarrow f_{q}^{q-1}\left(\tilde{x}_{q}\right)=\int_{\Omega} \frac{f_{q}(y)}{\left|\tilde{x}_{q}-y\right|^{n-\alpha}} d y+\lambda \int_{\Omega} \frac{f_{q}(y)}{\left|\tilde{x}_{q}-y\right|^{n-\alpha-1}} d y \leq C<\infty
$$

as $q \rightarrow\left(q_{\alpha}\right)^{-}$, which gives a contradiction.

Now we are left to prove claim (4.5).

We first notice that

$$
Q_{\lambda, q}(\Omega)=Q_{\lambda, q}(\Omega) h_{q}(0)=\int_{\Omega_{\mu}} \frac{h_{q}^{p-1}(y)}{|y|^{n-\alpha}}\left(1+\lambda \mu_{q}|y|\right) d y .
$$

Thus,

$$
\begin{aligned}
\int_{\Omega_{\mu}} h_{q}^{p-1}(y)|y|^{\alpha-n} d y & =\frac{1}{(1-|\lambda| d(\Omega))} \int_{\Omega_{\mu}} h_{q}^{p-1}(y)|y|^{\alpha-n}(1-|\lambda| d(\Omega)) d y \\
& \leq \frac{1}{(1-|\lambda| d(\Omega))} \int_{\Omega_{\mu}} h_{q}^{p-1}(y)|y|^{\alpha-n}\left(1+\lambda \mu_{q}|y|\right) d y \\
& \leq C<\infty,
\end{aligned}
$$

uniformly as $q \rightarrow\left(q_{\alpha}\right)^{-}$. Since $h_{q} \geq 1$ and $p<0$, we have

$$
\int_{\Omega_{\mu}} h_{q}^{p-1}(y) d y \leq C<\infty
$$

uniformly as $q \rightarrow\left(q_{\alpha}\right)^{-}$. 
On the other hand, we also have

$$
\int_{\Omega_{\mu}} h_{q}^{p-1}(y) d y \geq c_{0}>0, \text { as } q \rightarrow\left(q_{\alpha}\right)^{-} .
$$

Otherwise, if there exists a sequence $q_{n} \rightarrow\left(q_{\alpha}\right)^{-}$such that $\int_{\Omega_{\mu}} h_{q_{n}}^{p_{n}-1}(y) d y \rightarrow 0$ with $\frac{1}{p_{n}}+\frac{1}{q_{n}}=1$, then for given $R_{0}>0$ and $\epsilon>0$ small we can take $R>>R_{0}$ large enough, such that for $z \in \Omega_{\mu} \cap B\left(0, R_{0}\right)$, as $q_{n}$ close to $\left(q_{\alpha}\right)^{-}$,

$$
\begin{aligned}
1 \leq & h_{q_{n}}(z) \\
= & \frac{1}{Q_{\lambda, q_{n}}(\Omega)}\left(\int_{\Omega_{\mu} \backslash B(0, R)} \frac{h_{q_{n}}^{p_{n}-1}(y)}{|z-y|^{n-\alpha}} d y+\int_{\Omega_{\mu} \cap B(0, R)} \frac{h_{q_{n}}^{p_{n}-1}(y)}{|z-y|^{n-\alpha}} d y\right. \\
& \left.+\lambda \mu_{q_{n}} \int_{\Omega_{\mu} \backslash B(0, R)} \frac{h_{q_{n}}^{p_{n}-1}(y)}{|z-y|^{n-\alpha-1}} d y+\lambda \mu_{q_{n}} \int_{\Omega_{\mu} \cap B(0, R)} \frac{h_{q_{n}}^{p_{n}-1}(y)}{|z-y|^{n-\alpha-1}} d y\right) \\
\leq & \frac{1}{Q_{\lambda, q_{n}}(\Omega)}\left(\int_{\Omega_{\mu} \backslash B(0, R)} \frac{h_{q_{n}}^{p_{n}-1}(y)}{|z-y|^{n-\alpha}} d y+\int_{\Omega_{\mu} \cap B(0, R)} \frac{h_{q_{n}}^{p_{n}-1}(y)}{|z-y|^{n-\alpha}} d y\right. \\
& \left.+\lambda \mu_{q_{n}} \int_{\Omega_{\mu} \backslash B(0, R)} \frac{h_{q_{n}}^{p_{n}-1}(y)}{|z-y|^{n-\alpha-1}} d y\right) \\
\leq & \frac{1}{Q_{\lambda, q_{n}}(\Omega)}\left(\left(1+\frac{R_{0}}{R}\right)^{\alpha-n} \int_{\Omega_{\mu} \backslash B(0, R)} \frac{h_{q_{n}}^{p_{n}-1}(y)}{|y|^{n-\alpha}} d y+\left(R+R_{0}\right)^{\alpha-n} \int_{\Omega_{\mu} \cap B(0, R)} h_{q_{n}}^{p_{n}-1}(y) d y\right. \\
& \left.+\lambda \mu_{q_{n}}\left(1-\frac{R_{0}}{R}\right)^{\alpha+1-n} \int_{\Omega_{\mu} \backslash B(0, R)} \frac{h_{q_{n}}^{p_{n}-1}(y)}{|y|^{n-\alpha-1}} d y\right) \\
= & \frac{1}{Q_{\lambda, q_{n}}(\Omega)}\left(\left(1+\frac{R_{0}}{R}\right)^{\alpha-n} \int_{\Omega_{\mu} \backslash B(0, R)} \frac{h_{q_{n}}^{p_{n}-1}(y)}{|y|^{n-\alpha}} d y+\left(R+R_{0}\right)^{\alpha-n} \int_{\Omega_{\mu} \cap B(0, R)} h_{q_{n}}^{p_{n}-1}(y) d y\right. \\
& \left.+\lambda \mu_{q_{n}}\left(1-\frac{R_{0}}{R}\right)^{\alpha+1-n} \int_{\Omega_{\mu}} \frac{h_{q_{n}}^{p_{n}-1}(y)}{|y|^{n-\alpha-1}} d y-\lambda \mu_{q_{n}}\left(1-\frac{R_{0}}{R}\right)^{\alpha+1-n} \int_{\Omega_{\mu} \cap B(0, R)} \frac{h_{q_{n}}^{p_{n}-1}(y)}{|y|^{n-\alpha-1}} d y\right) \\
\leq & \frac{1}{Q_{\lambda, q_{n}}(\Omega)}\left(\left(1+\frac{R_{0}}{R}\right)^{\alpha-n} \int_{\Omega_{\mu}} \frac{h_{q_{n}}^{p_{n}-1}(y)}{|y|^{n-\alpha}} d y+\lambda \mu_{q_{n}}\left(1-\frac{R_{0}}{R}\right)^{\alpha+1-n} \int_{\Omega_{\mu}} \frac{h_{q_{n}}^{p_{n}-1}(y)}{|y|^{n-\alpha-1}} d y\right) \\
& +\left(R+R_{0}\right)^{\alpha-n} \frac{1}{Q_{\lambda, q_{n}}(\Omega)} \int_{\Omega_{\mu}} h_{q_{n}}^{p_{n}-1}(y) d y-\lambda \mu_{q_{n}}\left(R-R_{0}\right)^{\alpha+1-n} \frac{1}{Q_{\lambda, q_{n}}(\Omega)} \int_{\Omega_{\mu}} h_{q_{n}}^{p_{n}-1}(y) d y \\
\leq & 1+\epsilon .
\end{aligned}
$$

That is, $h_{q_{n}}(z) \rightarrow 1, z \in \Omega_{\mu} \cap B\left(0, R_{0}\right)$ uniformly as $q_{n} \rightarrow\left(q_{\alpha}\right)^{-}$. Then for $R_{0}>0$ large, since $\int_{\Omega_{\mu} \cap B\left(0, R_{0}\right)} h_{q_{n}}^{p_{n}-1}(y) d y \leq \int_{\Omega_{\mu}} h_{q_{n}}^{p_{n}-1}(y) d y$ and $\Omega_{\mu}$ goes to either $\mathbb{R}_{T}^{n}:=\left\{\left(z_{1}, z_{2}, \cdots, z_{n}\right) \mid z_{n}>T \geq 0\right\}$ or $\mathbb{R}^{n}$, we obtain a contradiction to (4.13).

By (4.4), we have

$$
\begin{aligned}
& \lim _{|z| \rightarrow \infty} \frac{1}{|z|^{\alpha-n}} \int_{\Omega_{\mu}} \frac{h_{q}^{p-1}(y)}{|z-y|^{n-\alpha}} d y \\
\geq & \lim _{|z| \rightarrow \infty} Q_{\lambda, q}(\Omega) \frac{h_{q}(z)}{|z|^{\alpha-n}}=\lim _{|z|^{\prime \rightarrow \infty}} \frac{\int_{\Omega_{\mu}} \frac{h_{q}^{p-1}(y)}{|z-y|^{n-\alpha}}\left(1+\lambda \mu_{q}|z-y|\right) d y}{|z|^{\alpha-n}} \\
\geq & (1-|\lambda| d(\Omega)) \lim _{|z| \rightarrow \infty} \frac{\int_{\Omega_{\mu}} \frac{h_{q}^{p-1}(y)}{|z-y|^{n-\alpha}} d y}{|z|^{\alpha-n}} .
\end{aligned}
$$


Since $\frac{1}{|z|^{\alpha-n}} \frac{h_{q}^{p-1}(y)}{|z-y|^{n-\alpha}} \leq 2^{\alpha-n} h_{q}^{p-1}(y)\left(1+|y|^{\alpha-n}\right)$ as $|z| \rightarrow \infty$, and $\int_{\Omega_{\mu}} h_{q}^{p-1}(y)(1+$ $\left.|y|^{\alpha-n}\right) d y \leq C$ by (4.12) and (4.13), we then have

$$
\lim _{|z| \rightarrow \infty} \frac{1}{|z|^{\alpha-n}} \int_{\Omega_{\mu}} \frac{h_{q}^{p-1}(y)}{|z-y|^{n-\alpha}} d y=\int_{\Omega_{\mu}} h_{q}^{p-1}(y) d y .
$$

Hence by (4.15), (4.16), (4.13) and (4.14) we obtain the claim (4.5). Hereby we complete the proof of Lemma 4.3 .

Proof of Proposition 4.2 Let $f_{q}>0$ being solutions to (4.1) for $q \in\left(0, q_{\alpha}\right)$, which are also the minimal energy functions to energy $Q_{\lambda, q}$. Then by Lemma 4.3 . we know that $\left\{f_{q}\right\}$ are uniformly bounded above and bounded below by a positive constant. Thus they are equicontinuous due to equation (4.1). It follows that $f_{q} \rightarrow f_{*}$ as $q \rightarrow\left(q_{\alpha}\right)^{-}$in $C(\bar{\Omega})$, and $f_{*}$ is the energy minimizer for $Q_{\lambda}$.

Completion of the Proof of Theorem 1.1. Lemma 4.1] and Proposition 4.2 implies the existence of a positive solution $f \in L^{p_{\alpha}}(\Omega) \cap C(\bar{\Omega})$ to the equation (1.1) for $q=\frac{2 n}{n+\alpha}, \lambda \in\left(-\frac{1}{d(\Omega)}, 0\right)$. It is also easy to see that $f \in C^{1}(\bar{\Omega})$.

\section{Acknowledgements}

We dedicate this paper to Professor Haïm Brezis to celebrate his seventy five birthday. We thank him for his great influence on us in the study of elliptic equations through his lectures and numerous papers, in particular, the paper with Louis Nirenberg [2. The project is partially supported by the National Natural Science Foundation of China (Grant No. 11571268) and the Fundamental Research Funds for the Central Universities (Grant No. GK201802015) and Simmons Collaboration (Grant No. 280487).

\section{REFERENCES}

[1] W. Beckner, Functionals for Multilinear Fractional Embedding, Acta Math. Sinica, English Series, 31 (2015), 1-28.

[2] H. Brezis, L. Nirenberg, Positive solutions of nonlinear elliptic equations involving critical Sobolev exponents Commun. Pure Appl. Math., 36 (1983), 437-477.

[3] W. Chen, Y. Fang, R.Yang, Semilinear equations involving the fractional Laplacian on domains, arXiv:1309.7499

[4] J. Dou, M. Zhu, Reversed Hardy-Littlewood-Sobolev inequality, Int. Math. Res. Not., 2015 (2015), 9696-9726.

[5] J. Dou, M. Zhu, Integral equations on bounded domains, J. Fun. Anal., (2018), https://doi.org/10.1016/j.jfa.2018.05.020.

[6] J. Dou, Q. Guo, M. Zhu, Subcritical approach to sharp Hardy-Littlewood-Sobolev type inequalities on the upper half space, Adv. Math., 312 (2017) 1-45; Corrigendum to "Subcritical approach to sharp Hardy-Littlewood-Sobolev type inequalities on the upper half space"[Adv. Math., 312 (2017) 1-45], Adv. Math., 317 (2017) 640-644.

[7] E. H. Lieb, Sharp constants in the Hardy-Littlewood-Sobolev and related inequalities, Ann. of Math., 118 (1983), 349-374.

[8] Q.N. Ngô, V.H. Nguyen, Sharp reversed Hardy-Littlewood-Sobolev inequality on $\mathbb{R}^{n}$, Israel J. Math., 220 (2017), 189-223.

Jingbo Dou, School of Mathematics and Information Science, Shaanxi Normal UniVersity, Xi'An, ShaAnXi, 710119, China

E-mail address: jbdou@snnu.edu.cn 
Qianqiao Guo, Department of Applied Mathematics, Northwestern Polytechnical University, Xi'An, ShaAnXi, 710129, China

E-mail address: gqianqiao@nwpu.edu.cn

Meijun Zhu, Department of Mathematics, The University of Oklahoma, Norman, OK 73019, USA

E-mail address: mzhu@math.ou.edu 